\title{
To Drain or Not to Drain? That Is the Question
}

\author{
Guido Costamagna $\cdot$ Ivo Boškoski
}

Published online: 19 March 2013

(c) Springer Science+Business Media New York 2013

The paper by Siddiqui et al. [1] reports on a retrospective, multicenter study of self-expandable metal stents (SEMS) for preoperative biliary decompression in patients with resectable and borderline resectable carcinoma of the pancreatic head. Two-hundred and forty-one patients (resectable 174, borderline resectable 67) were evaluated. The primary outcome was the effectiveness of SEMS for adequate biliary decompression; secondary outcomes were safety of SEMS in preoperative patients and patency rates and long-term clinical outcomes. Patients with T4 disease were also included because some studies have reported that neoadjuvant therapy downstages tumors, enabling safe tumor margin-negative resection [2-4]. The overall survival was $49 \%$ at 27 months. The authors concluded that SEMS should be considered for patients with resectable or borderline resectable pancreatic cancer with the initial finding of obstructive jaundice, particularly if neoadjuvant therapy is planned. Although retrospective, this study is unique because it shows that SEMS are safe and effective in achieving durable biliary drainage in patients with pancreatic cancer receiving neoadjuvant therapy.

In patients who are candidates for neoadjuvant therapy, initial biliary drainage is advisable since neoadjuvant therapy can take up to several months to accomplish prior to surgery [5]. Biliary drainage before neoadjuvant therapy gives symptomatic relief, reduces the risk of cholangitis due to chemotherapy-related immunosuppression (although cholangitis is rare in malignant biliary obstruction in patients with an intact papilla), prevents coagulopathy, and normalizes liver function tests. Biliary drainage might not

G. Costamagna $\cdot$ I. Boškoski $(\bowtie)$

Digestive Endoscopy Unit, Catholic University of Rome, Largo A. Gemelli, 8, 00168 Rome, Italy

e-mail: ivoboskoski@yahoo.com be necessary if patients are candidates for early surgery, and, in contrast, in some cases might even be harmful. Preoperative biliary drainage with plastic stents is associated with a higher rate of complications than in non-stented subjects [6]. Cholangitis in stented patients can delay surgery due to interruptions in therapy, increasing the probability of disease progression.

In the last two decades, SEMS, in comparison with plastic stents, have been inserted more frequently for benign and malignant biliary strictures. SEMS are superior to plastic stents due to their larger diameter, facilitating preoperative biliary decompression, coupled with a lower incidence of complications such as cholangitis $[7,8]$, but are much more expensive, limiting their use. However, plastic stents can be placed without the need for a tissue diagnosis, and are inexpensive. Nevertheless, placing a metal stent during the first drainage is advantageous over plastic since the drainage is more effective and durable, obviating the need for exchange in the case of nonresectability. It is our practice to obtain tissue when placing SEMS either by brushing, biopsies, or endoscopic ultrasound-guided fine needle aspiration.

In a recent prospective study by Aadam et al. [9], SEMS were placed in 55 patients with resectable and borderline resectable pancreatic head adenocarcinoma complicated by biliary obstruction. Anticipating surgical resection, the minimum length stent was used in order to preserve the maximum length of uninstrumented common hepatic duct above the stent, for future anastomosis. Of the 55 patients, 23 were resectable and 32 were borderline. SEMS malfunction occurred 260 days after placement in $15 \%$ of the patients (occlusion in $13 \%$ and migration in $2 \%$ ). SEMS malfunction occurred in $11 \%$ of patients who ultimately underwent pancreaticoduodenectomy and in $24 \%$ of patients with disease progression. Covered SEMS were 
placed in only 9 patients with previous cholecystectomy, without subsequent complications. The authors concluded that the presence of SEMS did not interfere with pancreaticoduodenectomy, and that removing SEMS is not necessary before surgery, if the shortest stent is used.

Conflicting data regarding the effect of preoperative biliary drainage and its relationship with perioperative complications has been published in the past decade [1013]. In our institution, the decision to stent is always made in conjunction with the involved specialists, such as the surgeons and oncologists, after tumor staging has occurred. The type of stent is chosen on the basis of tumor resectability, the preferences of the surgeon, and the eventuality of neoadjuvant therapy. For non-resectable tumors, predicted life expectancy and the possibility of performing adjuvant chemoradiotherapy are the main factors.

Due to the rapid progression of malignant disease, "quick and appropriate" diagnosis and staging is essential. Identifying patients early as candidates for neoadjuvant treatments is important because they may increase the probability of having negative surgical margins and reduce the number of micrometastases [14]. The European Society of Gastrointestinal Endoscopy recommends preoperative biliary drainage for patients with potentially resectable obstructive biliary malignancy who are candidates for neoadjuvant therapies, in those with acute cholangitis, or in patients with intense pruritus in which surgery has been delayed for other reasons [15]. Regarding the type of stent, it is preferable to use SEMS in patients who are candidates for neoadjuvant therapies, since plastic stents are more prone to complications. Uncovered metal stents are preferred over covered stents, since covered SEMS are at higher risk inducing cholecystitis, migration, and more rarely pancreatitis [16-19]. Furthermore, given the experience of Aadam et al. [9], SEMS should be chosen to be as short as possible in order to leave enough tissue for further anastomosis. Preliminary data show that drug-eluting metal stents appear to be safe, although long-term safety and efficacy data are lacking [20].

With the hope that the future will bring more sophisticated and effective approach and cure for these patients, it must be recalled that radical surgery is today the only potential cure for pancreatic cancer [21], and therefore maximal efforts should be done to bring the operable patient in the operating room as soon as possible.

\section{References}

1. Siddiqui AA, Mehendiratta V, Loren D, et al. Self-expanding metal stents (SEMS) for preoperative biliary decompression in patients with resectable and borderline-resectable pancreatic cancer: outcomes in 241 patients. Dig Dis Sci. (Epub ahead of print). doi:10.1007/s10620-012-2482-z.

2. Jessup JM, Steele G Jr, Mayer RJ, et al. Neoadjuvant therapy for unresectable pancreatic adenocarcinoma. Arch Surg. 1993;128: $559-564$.

3. Barugola G, Partelli S, Crippa S, et al. Outcomes after resection of locally advanced or borderline resectable pancreatic cancer after neoadjuvant therapy. Am J Surg. 2012;203:132-139.

4. McClaine RJ, Lowy AM, Sussman JJ, et al. Neoadjuvant therapy may lead to successful surgical resection and improved survival in patients with borderline resectable pancreatic cancer. $H P B$ (Oxf). 2010;12:73-79.

5. Varadhachary GR, Wolff RA, Crane $\mathrm{CH}$, et al. Preoperative gemcitabine and cisplatin followed by gemcitabine-based chemoradiation for resectable adenocarcinoma of the pancreatic head. J Clin Oncol. 2008;26:3487-3495.

6. van der Gaag NA, Rauws EA, van Eijck $\mathrm{CH}$, et al. Preoperative biliary drainage for cancer of the head of the pancreas. $N$ Engl $J$ Med. 2010;362:129-137.

7. Decker C, Christein JD, Phadnis MA, et al. Biliary metal stents are superior to plastic stents for preoperative biliary decompression in pancreatic cancer. Surg Endosc. 2011;25:2364-2367.

8. Wasan SM, Ross WA, Staerkel GA, et al. Use of expandable metallic biliary stents in resectable pancreatic cancer. Am J Gastroenterol. 2005;100:2056-2061.

9. Aadam AA, Evans DB, Khan A, et al. Efficacy and safety of selfexpandable metal stents for biliary decompression in patients receiving neoadjuvant therapy for pancreatic cancer: a prospective study. Gastrointest Endosc. 2012;76:67-75.

10. Sewnath ME, Karsten TM, Prins MH, et al. A meta-analysis on the efficacy of preoperative biliary drainage for tumors causing obstructive jaundice. Ann Surg. 2002;236:17-27.

11. Hoffman JP, Lipsitz S, Pisansky T, et al. Phase II trial of preoperative radiation therapy and chemotherapy for patients with localized, resectable adenocarcinoma of the pancreas: an Eastern Cooperative Oncology Group Study. J Clin Oncol. 1998;16:317323.

12. Pisters PW, Hudec WA, Hess KR, et al. Effect of preoperative biliary decompression on pancreaticoduodenectomy-associated morbidity in 300 consecutive patients. Ann Surg. 2001;234: 47-55.

13. Morris-Stiff G, Tamijmarane A, Tan YM, et al. Pre-operative stenting is associated with a higher prevalence of post-operative complications following pancreatoduodenectomy. Int J Surg. 2011;9:145-149.

14. Spitz FR, Abbruzzese JL, Lee JE, et al. Preoperative and postoperative chemoradiation strategies in patients treated with pancreaticoduodenectomy for adenocarcinoma of the pancreas. $J$ Clin Oncol. 1997;15:928-937.

15. Dumonceau JM, Tringali A, Blero D, et al. Biliary stenting: indications, choice of stents and results: European Society of Gastrointestinal Endoscopy (ESGE) clinical guideline. Endoscopy. 2012;44:277-298.

16. Isayama H, Komatsu Y, Tsujino $\mathrm{T}$, et al. Polyurethane-covered metal stent for management of distal malignant biliary obstruction. Gastrointest Endosc. 2002;55:366-370.

17. Isayama $\mathrm{H}$, Komatsu $\mathrm{Y}$, Tsujino $\mathrm{T}$, et al. A prospective randomised study of "covered" versus "uncovered" diamond stents for the management of distal malignant biliary obstruction. Gut. 2004;53:729-734.

18. Isayama H, Kawabe T, Nakai Y, et al. Cholecystitis after metallic stent placement in patients with malignant distal biliary obstruction. Clin Gastroenterol Hepatol. 2006;4:1148-1153.

19. Kahaleh M, Tokar J, Conaway MR, et al. Efficacy and complications of covered Wallstents in malignant distal biliary obstruction. Gastrointest Endosc. 2005;61:528-533. 
20. Shah T. Drug-eluting stents in malignant biliary obstruction: where do we stand? Dig Dis Sci. (Epub ahead of print). doi: 10.1007/s10620-012-2507-7.

21. Pancreatic Section of the British Society of Gastroenterology, Pancreatic Society of Great Britain and Ireland, Association of
Upper Gastrointestinal Surgeons of Great Britain and Ireland, Royal College of Pathologists, Special Interest Group for GastroIntestinal Radiology. Guidelines for the management of patients with pancreatic cancer periampullary and ampullary carcinomas. Gut. 2005;54:v1-v16. 